\title{
The infrared optical absorption spectra of the functionalized nanocrystalline diamond surface
}

\author{
Z. Remes ${ }^{\text {a,* }}$, A. Kromka ${ }^{\text {a }}$, H. Kozak ${ }^{\text {a }}$, M. Vanecek ${ }^{\text {a }}$, K. Haenen ${ }^{\text {b }}$, S. Wenmackers ${ }^{\text {b }}$ \\ a Institute of Physics of the AS CR, v. v. i., Cukrovarnicka 10, CZ-16200 Praha 6, Czech Republic \\ ${ }^{\mathrm{b}}$ Hasselt University, Institute for Materials Research (IMO), Wetenschapspark 1, B-3590 Diepenbeek, Belgium
}

\section{A R T I C L E I N F O}

Available online 3 December 2008

\section{Keywords:}

Nanocrystalline diamond

Photochemical functionalization

Spin coating

Polymer

Infrared spectroscopy

Fluorescence microscopy

\begin{abstract}
A B S T R A C T
We propose a new method of detecting the functional groups at the NCD surface based on the interfrencefree infrared reflection absorption spectroscopy of p-polarized IR light at Brewster's angle of incidence (BAIRRAS). We report IR absorbance spectra of a linker molecule monolayer (10-undecenoic acid) covalently bonded to the NCD surface with and without DNA fragments coupled to it, and IR spectra of organosilane polymer coatings deposited on NCD surface by the spin coating technology. The homogeneity of the surface coatings was monitored by fluorescence microscopy.
\end{abstract}

(c) 2008 Elsevier B.V. All rights reserved.

\section{Introduction}

Low cost insulating (nominally undoped) and semiconducting (boron-doped) nanocrystalline diamond (NCD) films deposited on a large area substrates offer the unique mechanical, electrical and optical properties, as well as biocompatibility, chemical inertness, high affinity for covalent bonding with specific organic molecules and the ability to make the substrate electrically insulating or conducting $[1,2]$. The biomolecules can be covalently immobilized on the diamond surface via suitable linker molecules [3]. The main role of the linker molecules is to provide a high density of docking sites (the functional groups such as hydroxyl $(-\mathrm{OH})$, carbonyl $(>\mathrm{C}=\mathrm{O})$, carboxyl $(-\mathrm{COOH})$ or amino-group $\left(-\mathrm{NH}_{2}\right)$ ) for the specific attachment of biomolecules, and, at the same time, to maintain a sufficiently low density of electronic defects at the semiconductor surface [4]. This makes the functionalized NCD-coated substrates attractive for applications such as cells growth [5], DNA immobilization [6] or label-free, in-vitro, realtime, diamond-based DNA sensors [7].

Depending on the application and the desired physical and chemical properties of the surface, different strategies for NCD functionalization have been demonstrated. The experiments by Wenmackers et al. $[8,6]$ demonstrated the repeated hybridization and denaturation after covalent immobilization of DNA on photochemically functionalized, hydrogenated NCD surfaces. Alternatively, the NCD surface can also be functionalized with several nm thick organosilane polymers $[9,10,11]$. In the organosilane polymer coatings a trivalent silane headgroup participates in multidirectional and interchangeable intra- and interlayer modes of coupling, both

\footnotetext{
* Corresponding author. Tel.: +420 220318516.

E-mail address: remes@fzu.cz (Z. Remes).
}

covalent and non-covalent (hydrogen bonds). Polymerization appears via rearranging $-\mathrm{Si}-\mathrm{O}-\mathrm{Si}-$ and $-\mathrm{Si}-\mathrm{OH}$ moieties into a $3 \mathrm{D}$ network [12].

The IR absorbance spectra provide quantification of surface monolayers and thin coatings and direct evidence for the appearance and disappearance of the specific functional groups in the course of a chemical reaction. However, multireflections in a transparent film such as NCD introduce interference fringes in the IR spectra, making the identification of functional groups difficult. We propose a new method of detecting the functional groups at the NCD surface based on the interfrence-free infrared reflection absorption spectroscopy of p-polarized IR light at Brewster's angle of incidence (BA-IRRAS). We report IR absorbance spectra of a linker molecule monolayer (10undecenoic acid, UA) covalently bonded to the NCD surface with and without DNA fragments coupled to it, and IR spectra of ultra-thin organosilane polymer coatings deposited on NCD surface by spin coating technology.

\section{Experimental}

The optically smooth NCD films of size about $10 \times 10 \mathrm{~mm}^{2}$ and $200 \mathrm{~nm}$ thick, were deposited in $2.45 \mathrm{GHz}$ plasma chemical vapor deposition (CVD) reactors on the one side polished $\mathrm{Si}$ wafer at the Institute of Physics of the ASCR, v.v.i., Prague, Czech Rep. [13,14] and at the Institute for Materials Research (IMO), Hasselt University, Belgium $[15,16]$. Prior to CVD growth, the Si substrates were seeded on the polished side by ultradispersed detonation nanodiamond powder yielding a very dense adsorbed diamond nanoparticle layer without damaging the polished Si surface.

Onto NCD samples prepared at IMO (\#1, \#2, \#3), organic linkers and short DNA fragments were covalently coupled, according to the 


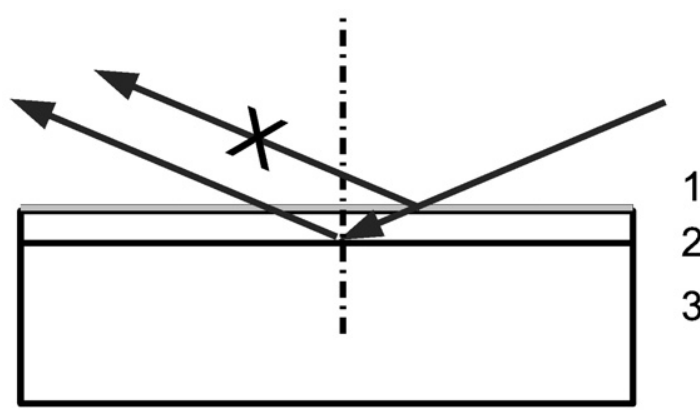

Fig. 1. The principle of the interference-free reflectance-absorbance spectroscopy of surface monolayer or ultra-thin coating (1) on optically smooth NCD film (2) deposited on one side polished Si substrate (3). The p-polarized IR light at Brewster's angle of incidence passes two times the functionalized NCD surface where part of the light is absorbed at wavelengths characteristic for the functional groups vibrations. The reflectance at $\mathrm{NCD}$ /air interface is negligible; the $\mathrm{NCD} / \mathrm{Si}$ interface reflects about $2 \%$ light. The bottom of $\mathrm{Si}$ substrate surface is optically rough and does not contribute to the reflected light.

method in [16]. First, all samples were cleaned in boiling mixture of $\mathrm{H}_{2} \mathrm{SO}_{4}$ with $\mathrm{KNO}_{3}$, rinsed several times in ultra-pure water in ultrasonic bath and on hot plate in turn, and hydrogenated in $\mathrm{H}-$ plasma. Second, the organic linker 10-undecenoic acid (UA) was covalently coupled to the surface, using photochemistry. Inside a nitrogen flushed glovebox, a droplet of $100 \mu \mathrm{l}$ UA $\left(\mathrm{C}_{11} \mathrm{H}_{20} \mathrm{O}_{2} 99 \%\right.$, Acros Organics, Geel, Belgium) was pipetted on the sample surface. On top of the UA-covered surface, a UV-transparent quartz microscope slide was placed, leaving only a thin film of UA on the surface. For $20 \mathrm{~h}$ the samples were placed at a distance of about $2 \mathrm{~cm}$ under an intense UV lamp (>2.5 $\mathrm{mW} \mathrm{cm}^{-2}$, "TUV G4T4 4W", Philips, Eindhoven, The Netherlands) mounted in an EPROM eraser box ("ME5", Lawtronics, Edenbridge, UK) to achieve a carboxyl-terminated surface. After this reaction, the samples are rinsed in pure acetic acid $\left(\mathrm{C}_{2} \mathrm{H}_{4} \mathrm{O}_{2} 99-100 \%\right.$ pure, Merck) at $100{ }^{\circ} \mathrm{C}$ (to remove non-covalently attached UA), followed by the same washing steps in ultra-pure water as after the chemical oxidation. The sample \#1 was kept like this. The other two samples (\#2 and \#3) were subsequently used for covalent coupling of amino-modified probe DNA to the carboxyl-terminated surface, using the zero-length crosslinker 1-ethyl-3-[3-dimethylaminopropyl]-carbodiimide (EDC) (Perbio Science, Erembodegem, Belgium). This was done in 2-[N-morpholino]-ethanesulphonic acid (MES) buffer (Perbio Science). The probe DNA was single stranded (ss), consisting of 36 bases (36b): 5'-NH2-C6H12-AAA-AAA-ACC-CCT-GCA-GCC-CAT-GTATAC-CCC-CGA-ACC-3' (Invitrogen, Merelbeke, Belgium). The NCD sample covered with 36b ssDNA is then allowed to hybridize to perfectly complementary target ssDNA of 29b: 5'-GGT-TCG-GGG-GTATAC-ATG-GGC-TGC-AGG-GG-3' (Invitrogen), carrying a fluorescent probe, Alexa Fluor ${ }^{\circledR} 488$, at its $5^{\prime}$ side. The result of the hybridization was 29 base pairs (bp) double stranded (ds) DNA connected to an A7ss-tail. This is obtained more precisely by incubating the samples with 600 pmol target ssDNA, in MicroHyb ${ }^{\mathrm{TM}}$ hybridization buffer (Invitrogen, Merelbeke, Belgium). The samples were placed in a closed container at $80^{\circ} \mathrm{C}$ for $2 \mathrm{~h}$, and, to avoid evaporation, a droplet of water was enclosed. After this, stringency washings were performed by rinsing the samples in saline sodium citrate (SSC) buffer containing $0.5 \%$ sodium dodecyl sulfate (SDS) for $30 \mathrm{~min}$ at room temperature (RT), followed by two rinses for $5 \mathrm{~min}$ in $0.2 \times \mathrm{SSC}$ buffer, at $75{ }^{\circ} \mathrm{C}$ and at RT, respectively. At this point, the successful attachment was confirmed with confocal fluorescence microscopy [16]. For IR measurements, the samples are dried directly from buffer (rinsing in ultra-pure water would remove the complementary strand) under a flow of N2. The sample \#2 (NCD-UA-29bpDNA) was kept like this. Shortly before FTIR measurements, the complementary strand was removed from sample \#3 (NCD-UA-36bDNA) by denaturation in boiling ultra-pure water (20 $\mathrm{min})$.
The NCD films grown in Prague were also cleaned in hot acid solution $\left(\mathrm{H}_{2} \mathrm{SO}_{4}+\mathrm{KNO}_{3}\right)$ and then boiling water. After cleaning, they were oxidized $3 \mathrm{~min}$ in the oxygen plasma (RF plasma, $300 \mathrm{~W}$ ) to enhance their hydrophilic character. The samples were blown off by clean nitrogen immediately before applying the polymer coatings to reduce the surface contamination by dust particles. The very thin polymer coatings were deposited by dropping a small amount of diluted organosilane coupling agent $\mathrm{N}$-(6-aminohexyl) aminopropyl trimethoxysilane (AHAPS) on the substrate followed by spin-coating at 8000 rotations per minute (rpm). The thickness of the coating was controlled by diluting AHAPS in toluene.

Reflectance-absorbance FTIR spectra were recorded at the Brewster's angle of incidence ( $67^{\circ}$ for diamond) using the Nicolet Nexus FTIR spectrometer equipped with a wire grid ZnSe polarizer, $\mathrm{KBr}$ beamsplitter, liquid nitrogen cooled MCT detector and variable angle reflectance accessory. The spectrometer was purged with dry air to reduce water-related absorption. The optical absorbance, A, was calculated in the standard absorbance units as $A=-\log \left(R / R_{0}\right)$ where $R$ is the reflectance measured with functionalized sample and $R_{0}$ the reference (background) spectrum of the substrate with clean surface. The reference $R_{0}$ spectra were recorded $15 \mathrm{~min}$ before and after the sample spectra to improve the reproducibility.

The inverted laser-scanning confocal fluorescence microscope set-up was a "Zeiss LSM 510 META" Axiovert 200 M from Carl Zeiss (NV/SA, Zaventem, Belgium). The wavelength of $488 \mathrm{~nm}$ of an Ar-ion laser was used for excitation; an additional 488/10 nm excitation interference filter was inserted before the excitation fibre optics to guarantee spectral purity. We applied a pinhole of $150 \mu \mathrm{m}$, a pixel time of $25.6 \mu \mathrm{s}$, and a band pass emission filter of 500 to $550 \mathrm{~nm}$. A LD-Achroplan $40 \times$ objective with 0.6 numerical aperture was used for imaging in air.

\section{Results and discussion}

Since p-polarized light is not reflected at the Brewster's angle of incidence at the diamond/air interface, the interference fringes in thin films are eliminated. The IR light is reflected only at the smooth film / substrate interface as it passes through the thin film, see Fig. 1. The reflectivity of the diamond/Si interface is only about $2 \%$, but still the optical absorbance down to $10^{-3}$ (in the standard absorbance units) can be detected, except in the spectral region $2200-2400 \mathrm{~cm}^{-1}$ where residual $\mathrm{CO}_{2}$ absorbs. This spectral region was removed from all

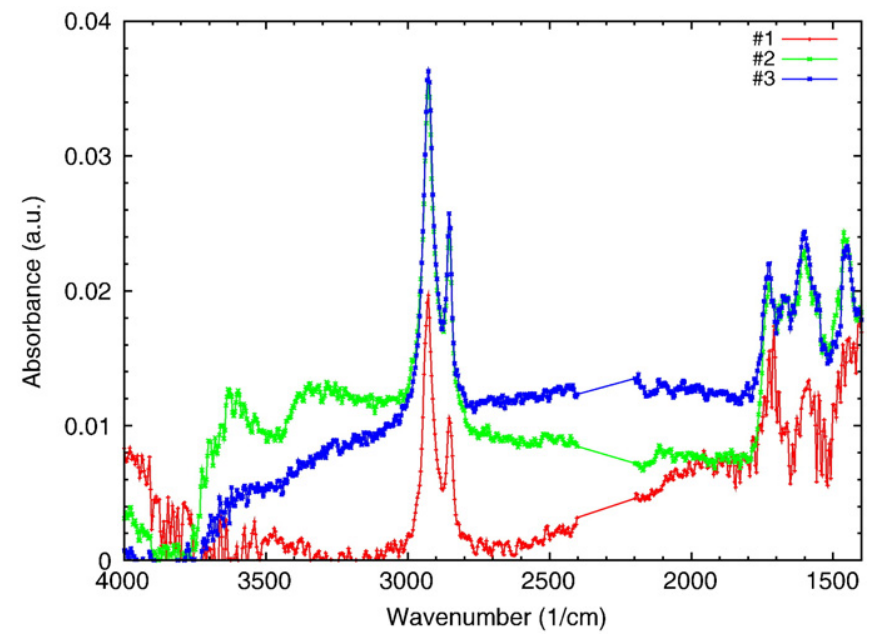

Fig. 2. IR absorbance spectra of a linker molecule monolayer (10-undecenoic acid (UA), sample \#1) covalently bonded to the NCD surface with and without covalently bonded DNA fragments (\#2 and \#3). The sample \#2 contains 29 base pairs double stranded DNA (NCD-UA-29bpDNA), and the sample \#3 single stranded DNA, consisting of 36 bases (NCD-UA-36bDNA). 


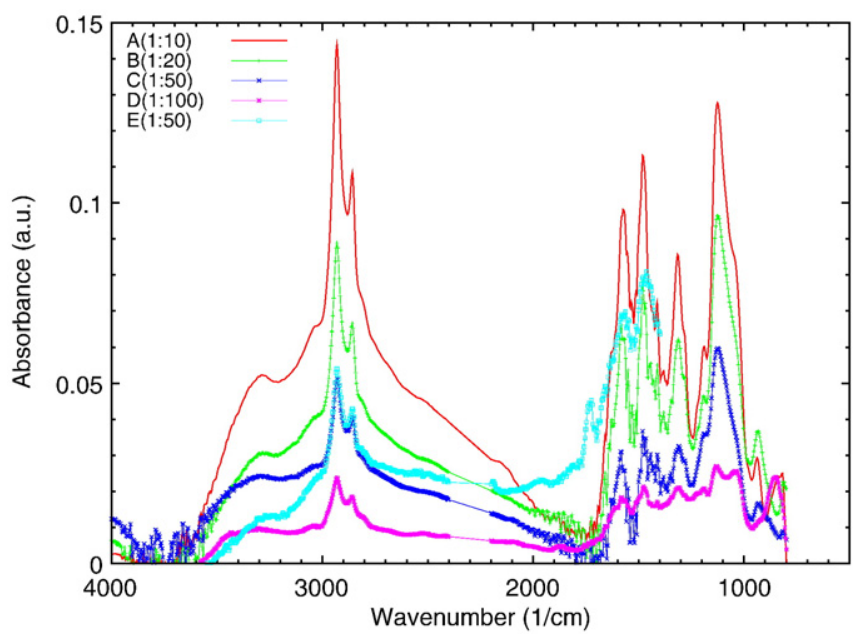

Fig. 3. Comparison of the FTIR absorbance spectra of organosilane (AHAPS) polymer films deposited by spin coating ( $8000 \mathrm{rpm}$ ) on Au (\#A-D) and NCD (\#E) substrates. The AHAPS film thickness was controlled by diluting AHAPS in toluene. The spin coating of not-diluted polymer leads to homogeneous film with thickness about $1 \mu \mathrm{m}$ and the optical absorbance spectra about $10 \times$ stronger then \#A (for clarity not shown in graph).

spectra. The IR spectra below $1400 \mathrm{~cm}^{-1}$ were not reproducible on NCD samples. A dry air purging was necessary to obtain reliable spectra in the spectral regions $1350-1900 \mathrm{~cm}^{-1}$ and $3500-3950 \mathrm{~cm}$ ${ }^{-1}$ where the background noise significantly increased when the residual gaseous water was not fully removed from spectrometer.

Fig. 2 shows the IR absorbance spectra of a linker molecule monolayer (10-undecenoic acid (UA), sample \#1) covalently bonded to the NCD surface with and without bonded DNA fragments (\#2 and \#3). We assign the absorbance peak at $1714 \mathrm{~cm}^{-1}$ to $v(C=0)$ (stretch vibration of carbonyl), and the peaks at $2852 \mathrm{~cm}^{-1}$ and $2921 \mathrm{~cm}^{-1}$ to $v_{\mathrm{s}}\left(\mathrm{C}-\mathrm{H}_{2}\right)$ and $v_{\mathrm{as}}\left(\mathrm{C}-\mathrm{H}_{2}\right)$ (symmetric and asymmetric stretch vibrations of $\left.\mathrm{C}-\mathrm{H}_{2}\right)$, respectively $[17,18]$. For the sample \#1 there are no peaks in the region of $3000-3150 \mathrm{~cm}^{-1}$, which would imply the presence of unsaturated hydrocarbons [19]. Thus, there is no indication of the presence of unreacted UA molecules after the cleaning in acetic acid. In the region of $3200-3570 \mathrm{~cm}^{-1}$, a peak due to the $\mathrm{O}-\mathrm{H}$ stretch is expected [19]: here a clear signal is absent too. Although the spectra have been collected down to a wavenumber of $800 \mathrm{~cm}^{-1}$, the results for wavenumbers below $1400 \mathrm{~cm}^{-1}$ are not shown in Fig. 2: this spectral region exhibits the one-phonon absorption of NCD. Since the $\mathrm{C}-\mathrm{O}$ stretch is expected in this range [19], the presence of this peak could not be verified.

Regarding the samples \#2 and \#3, we note that the peaks at $2852 \mathrm{~cm}^{-1}$ and $2921 \mathrm{~cm}^{-1}$, assigned to the saturated hydrocarbons of the UA-chain, are essentially unchanged; this is to be expected, since only the end-group of the UA molecule is involved in the coupling to DNA. The peak at $1714 \mathrm{~cm}^{-1}$, assigned to $v(C=0)$, is less clear in the spectra of samples with DNA as compared to sample with only the UA, probably overlapping with other peaks related to groups in the DNA (and possibly a shift of the $\mathrm{C}=\mathrm{O}$-related peak). In the involved wavenumber region, delocalised vibration modes are indeed expected in DNA $[20,21]$. The modes in the $1550-1750 \mathrm{~cm}^{-1}$ range are attributed primarily to $\mathrm{C}=\mathrm{O}$ stretching and $\mathrm{N}-\mathrm{H} 2$ bending vibrations in the DNA bases [22]. In the spectra of samples \#2 and \#3, we attribute the absorbance peak at $1692 \mathrm{~cm}^{-1}$ to 'Amide I' [23]. Because we lack information below $1400 \mathrm{~cm}^{-1}$, we were not able to confirm the presence of DNA-related peaks at $1550 \mathrm{~cm}^{-1}$ (due to 'Amide II' [23]), or at $1220 \mathrm{~cm}^{-1}$ and $1050 \mathrm{~cm}^{-1}$ (attributed to stretching vibrations of the $\mathrm{PO}_{2}^{-}$in the DNA backbone $[23,24]$ ). IR spectra can in principle be used to distinguish between DNA in single and double stranded form: according to [22], the influence of hybridization on the $\mathrm{C}=\mathrm{O}$ stretching and $\mathrm{N}-\mathrm{H}_{2}$ bending vibrations due to Watson-Crick $\mathrm{H}-$ bonds can be observed in the $1550-1750 \mathrm{~cm}^{-1}$ range. However, the spectra in Fig. 2 are not decisive on this point. A possible explanation is that after drying of the surface, boiling the sample in ultra-pure water is not effective in removing of the complementary strands. Two additional difficulties encountered here, are the limited spectral range (due to absorptions in NCD below $1400 \mathrm{~cm}^{-1}$ ) and the overall low signal. Two weak peaks observed in the region $3250-3600 \mathrm{~cm}^{-1}$ may be attributed to amino and hydroxyl groups [19], both present in the DNA bases. These peaks are more pronounced in the NCD-UA29bpDNA (\#2) spectrum than in the NCD-UA-36bDNA (\#3) case, which is to be expected since more bases are present in the ds case than in the ss case.

The IR absorbance spectra can be used to estimate thickness of the surface coating. First, we applied spin coating technology to deposit AHAPS polymer coating (not yet diluted in toluene) on Au substrate (100 nm Au layer evaporated on glass substrate), and found the layer very homogeneous with thickness about $1 \mu \mathrm{m}$ (deduced from the optical interference fringes). The intensity of the absorbance peaks at $2852 \mathrm{~cm}^{-1}$ and $2921 \mathrm{~cm}^{-1}$ was in the order of 1 . The Au substrate was

A

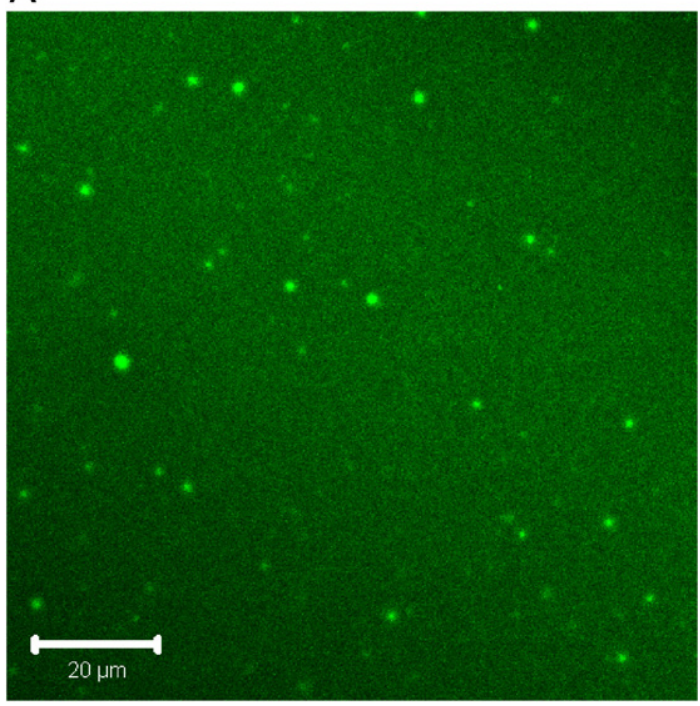

B

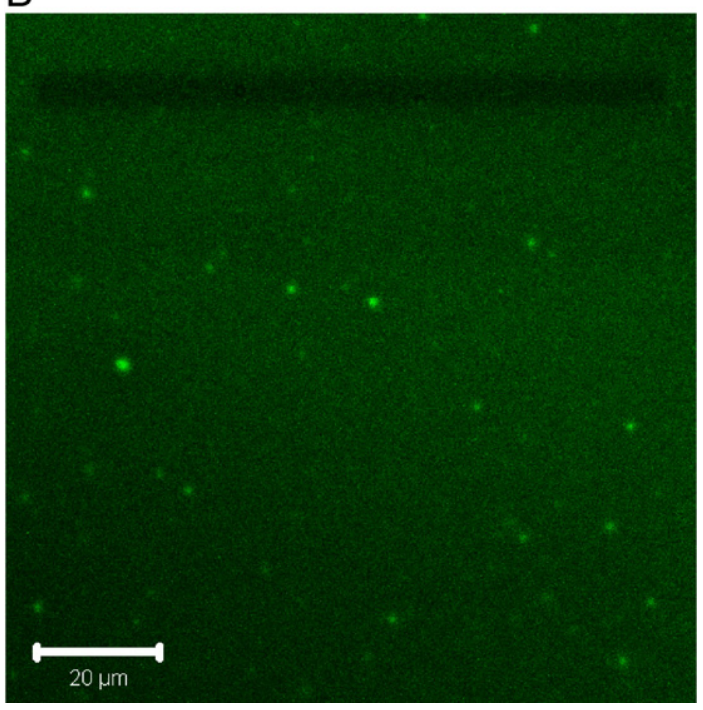

Fig. 4. Fluorescence images of NCD coated by AHAPS-based organosilane polymer (spin coating technology, AHAPS in toluene dilution 1:50) before (A) and after (B) photobleaching. The dark box in the upper part of the figure (B) corresponds to the area photobleached by the maximum laser intensity. 
used as a substrate because it allows detecting absorbance spectra in wide spectral range $\left(4000-800 \mathrm{~cm}^{-1}\right)$ and also because the reflectance at diamond Brewster's angle of incidence is not interference-free when the surface polymer coating is thick. Fig. 3 shows that the intensity of absorbance peaks at $2852 \mathrm{~cm}^{-1}$ and $2921 \mathrm{~cm}^{-1}$ decreases proportionally with dilution of AHAPS in toluene being related to the total amount of polymer mass deposited on the surface. From here we estimate the polymer coating thickness just few nm using the dilution 1:200. The carbonyl peak at $1720 \mathrm{~cm}^{-1}$ appeared in the absorbance spectra of the polymer on NCD in addition to the peaks observed in polymer on Au substrate. The origin of the carbonyl peak is not clear. Unfortunately, the FTIR spectra were not well reproducible and the fluorescence images revealed insulated polymer clusters, see Fig. 4A. The clusters appear as spots which emit fluorescence and there seems to be a continuous, fluorescing background. After photobleaching, see Fig. 4B, spots as well as background became dark (photo-bleached). This may imply that the NCD surface is indeed covered with a continuous polymer film or with a dense layer of polymer clusters smaller than the optical resolution.

\section{Conclusions}

The IR absorbance spectra were measured by the interference-free reflectance-absorbance FTIR spectroscopy at Brewster's angle. We have shown that FTIR absorbance spectroscopy can detect organic monolayers and coatings on NCD films down to few nm thickness and that the absorbance scales with the total mass of the organic molecules at the surface. In this paper we show for the first time the FTIR spectra of NCD surfaces functionalized covalently by a photochemically introduced 10 -undecenoic acid monolayer with and without bonded DNA fragments, and IR spectra of organosilane polymer coatings deposited on NCD surfaces by spin coating technology to achieve continuous and stable surface functionalization. The photochemical functionalization (which takes about $20 \mathrm{~h}$ ) can eventually be replaced by spin coating technology that takes just few minutes. In addition to the thin, continuous polymer coating, the polymer clusters have been also detected on the NCD surface coated by spin coating technology and these inhomogeneities need to be eliminated for further applications.

\section{Acknowledgement}

We thank Prof. Marcel Ameloot and Dr. Martin vandeVen of the microfluorimetry group, BIOMED, UHasselt, Belgium for the help with fluorescence microscopy, Dr. Veronique Vermeeren of BIOMED, UHasselt for DNA attachment to NCD and Dr. Katy Roodenko of ISAS,
Berlin for the help with interpretation of IR spectra of UA and DNA. This work was supported by Institutional Research Plan No. AV0Z10100521, the Grant Agency of the Academy of Sciences of the Czech Republic under contract KJB100100623, LC 510 Center of nanotechnology and DRIVE project (Diamond Research on Interfaces and Versatile Electronics) Marie Curie Research Training Network, contract MRTN-CT-2004-512224.

\section{References}

[1] D.M. Gruen, Annu. Rev. Mater. Sci. 29 (1999) 211.

[2] A. Hartl, E. Schmich, J.A. Garrido, J. Hernando, S.C.R. Catharino, S. Walter, P. Feulner, A. Kromka, D. Steinmuller, M. Stutzmann, Nat. Mater. 3 (2004) 736

[3] W. Yang, O. Auciello, J.E. Butler, W. Cai, J.A. Carlisle, J.E. Gerbi, D.M. Gruen, T. Knickerbocker, T.L. Lasseter, J.N. Russell Jr., L.M. Smith, R.J. Hamers, Nat. Mater. 1 (2002) 253.

[4] M. Stutzmann, J.A. Garrido, M. Eickhoff, M.S. Brandt, Phys. Stat. Sol. (A) 203 (2006) 3424.

[5] L. Grausova, A. Kromka, L. Bacakova, S. Potocky, M. Vanecek, V. Lisa, Diamond Relat. Mater. 17 (2008) 1405

[6] P. Christiaens, V. Vermeeren, S. Wenmackers, M. Daenen, K. Haenen, M. Nesládek, M. vandeVen, M. Ameloot, L. Michiels, P. Wagner, Biosens. Bioelectron. 22 (2006) 170.

[7] V. Vermeeren, N. Bijnens, S. Wenmackers, M. Daenen, K. Haenen, O.A. Williams, M. Ameloot, A. Vandeven, P. Wagner, L. Michiels, Langmuir 23 (2007) 13193.

[8] S. Wenmackers, P. Christiaens, M. Daenen, K. Haenen, M. Nesládek, M. van deVen, V. Vermeeren, L. Michiels, M. Ameloot, P. Wagner, Phys. Stat. Sol. (A) 202 (2005) 2212.

[9] Z. Remes, A. Choukourov, J. Stuchlik, J. Potmesil, M. Vanecek, Diamond Relat. Mater. 15 (2006) 745.

[10] Z. Remes, A. Kromka, M. Vanecek, A. Grinevich, H. Hartmannova, S. Kmoch, Diamond Relat. Mater. 16 (2007) 671.

[11] J. Hernando, T. Pourrostami, J.A. Garrido, O.A. Williams, D.M. Gruen, A. Kromka, D. Steinmüller, M. Stutzmann, Diamond Relat. Mater. 16 (2007) 138.

[12] K. Wen, R. Maoz, H. Cohen, J. Sagiv, A. Gibaud, A. Desert, B.M. Ocko, ACSNano 2 (2008) 579.

[13] V. Mortet, A. Kromka, R. Kravets, J. Rosa, V. Vorlicek, J. Zemek, M. Vanecek, Diamond Relat. Mater. 13 (2004) 604.

[14] S. Potocky, A. Kromka, J. Potmesil, Z. Remes, V. Vorlicek, M. Vanecek, M. Michalka, Diamond Relat. Mater. 16 (2007) 744

[15] O.A. Williams, O. Douheret, M. Daenen, K. Haenen, E. Osawa, M. Takahashi, Chem. Phys. Lett. 445 (2007) 255.

[16] V. Vermeeren, S. Wenmackers, M. Daenen, K. Haenen, O.A. Williams, M. Ameloot, M. vandeVen, P. Wagner, L. Michiels, Langmuir 16 (2008) 9125

[17] Y.L. Li, R. Tero, T. Nagasawa, T. Nagata, T. Urisu, Appl. Surf. Sci. 238 (2004) 238

[18] H.-B. Liu, S.-J. Xiao, Y.-Q. Chen, J. Chao, J. Wang, Y. Wang, Y. Pan, X. Z You, Z.-Z. Gu, J. Phys. Chem. B. Lett. 110 (2006) 17702

[19] J. Coates, in: R.A. Meyers (Ed.), in Encyclopedia of analytical chemistry, Wiley, Chichester, 2000.

[20] M. Banyay, M. Sarkar, A. Gräslund, Biophys. Chem. 104 (2003) 477.

[21] C. Lee, K.H. Park, M. Cho, J. Chem. Phys. 125 (2006) 114508.

[22] S. Moses, S.H. Brewer, L.B. Lowe, S.E. Lappi, L.B. Gilvey, M. Sauthier, R.C. Tenent, D.L. Feldheim, S. Franzen, Langmuir 20 (2004) 11134.

[23] S.B. Dev, L. Walters, Biopolymers 22 (1990) 289.

[24] K. Nakano, H. Matsunaga, K. Sai, N. Soh, T. Imato, Anal. Chim. Acta 578 (2006) 93. 